\title{
Tensile Behavior of Polyetheretherketone over a Wide Range of Strain Rates
}

\author{
Zakaria El-Qoubaa ${ }^{1}$ and Ramzi Othman ${ }^{2}$ \\ ${ }^{1}$ Institut de Recherche en Génie Civil et Mécanique, UMR CNRS 6183, École Centrale de Nantes, L'UNAM, 1 rue de la Noe, \\ BP 92101, 44321 Nantes Cedex 3, France \\ ${ }^{2}$ Mechanical Engineering Department, Faculty of Engineering, King Abdulaziz University, P.O. Box 80248, Jeddah 21589, Saudi Arabia \\ Correspondence should be addressed to Ramzi Othman; rothman1@kau.edu.sa
}

Received 13 March 2015; Accepted 24 May 2015

Academic Editor: Jan-Chan Huang

Copyright (C) 2015 Z. El-Qoubaa and R. Othman. This is an open access article distributed under the Creative Commons Attribution License, which permits unrestricted use, distribution, and reproduction in any medium, provided the original work is properly cited.

\begin{abstract}
Polyetheretherketone (PEEK) is used in several engineering applications where it has to bear impact loads. Nevertheless, the tensile behavior has only been studied in the quasi-static range of loading rates. To address the lack of data in the impact strain rate range, the tensile mechanical behavior of PEEK is investigated at room temperature over a large range of strain rates (from 0.001 to $1000 / \mathrm{s}$ ). The macroscopic volume change is studied under uniaxial tension using digital image correlation (DIC) method, showing a significant dilatation that reaches $16 \%$ at a logarithmic axial strain of $40 \%$. The true stress-strain behavior is therefore established based on the measured volume change. Elsewhere, the yield stress shows a significant sensitivity to strain rate. Besides, a new constitutive equation is proposed to take into account the increase in strain rate sensitivity at high strain rates. It assumes an apparent activation volume which decreases as the strain rate increases. The new constitutive equation gives similar results when compared to the Ree-Eyring equation. However, only three material constants are to be identified and are physically interpreted.
\end{abstract}

\section{Introduction}

Thanks to its high chemical and physical properties, polyetheretherketone (PEEK) is nowadays regarded as one of the most efficient thermoplastics. PEEK is widely used as a matrix in composites within several industrial applications, specifically, in automotive and aeronautic industries. Besides, PEEK can be used as a medical implant due to its biocompatibility $[1,2]$. PEEK's behavior depends on strain rate and temperature as well. Because of the existence of thermal transitions in polymers behavior, these two experimental parameters affect significantly the relaxation phenomena. In fact, studying the sensitivity to the strain rate, the crystallinity and the temperature contribute effectively to the enhancement of numerical modeling. Elsewhere, researchers, when testing polymers, have constantly assumed the incompressibility hypothesis regarding the evolution of the cross-sectional area, in order to characterize the true stress-strain. Actually, this assumption is more suitable for metals than polymers. As a consequence, it is of major importance to check the volume change of PEEK.
The strain rate sensitivity of PEEK's behavior was extensively studied under compression [3-9]. Walley and Field [3] have characterized the compressive yield stress of PEEK from 0.001 to $18000 / \mathrm{s}$. They showed that the yield stress increases linearly with increasing strain rate up to 2000/s. However, it drops drastically at very high stain rates. Hamdan and Swallowe [4] reported that strain rate sensitivity is almost independent of temperature, over the strain rate range from 0.001 to $1000 / \mathrm{s}$ and when temperature is lower than the glass transition. The strain rate sensitivity is about $4 \mathrm{MPa}$ /decade. Hamdan et al. $[5,6]$ have also showed that the cold-crystallization influences significantly the yield process for experiments undertaken above glass transition. Rae et al. [7] investigated the PEEK's yield stress over a strain rate range going from 0.0001 to $3000 / \mathrm{s}$ and a temperature range going from $-50^{\circ}$ to $150^{\circ} \mathrm{C}$. They reported an increase in the strain rate sensitivity at high strain rates. Recently, the authors [8] have showed that the compressive plastic deformation of PEEK is isochoric over a large range of strain rates and temperatures. They have also studied the compressive yield stress over a strain rate range of $0.0001-10000 / \mathrm{s}$ [9], reporting 


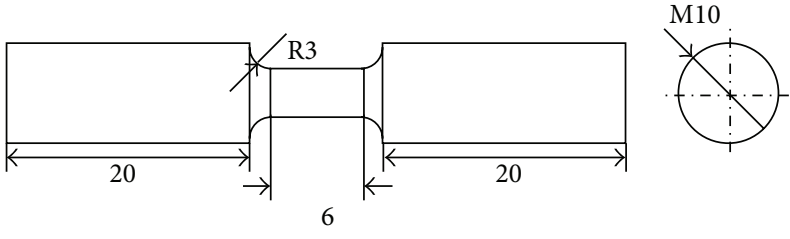

FIGURE 1: Specimen geometry (dimensions in $\mathrm{mm}$ ).

an increase in the strain rate sensitivity of the yield stress as the strain rate increases.

Contrary to the compressive behavior, the sensitivity of the PEEK's tensile behavior to the strain rate has not been reported in the open literature, to the best of the authors' knowledge. Indeed, all works that dealt with the PEEK's tensile properties have been limited to quasi-static strain rates [7, 10-13]. Rae et al. [7] have reported tensile stressstrain curves for strain rates lower than $0.02 /$ s. Lee et al. [11] showed that tensile properties are not affected by the change of crystallinity. On the contrary, it was reported in [12] that the yield stress increases when the crystallinity increases, whereas G'sell and Dahoun [13] investigated PEEK's response (at $180^{\circ} \mathrm{C}$ and $0.0005 / \mathrm{s}$ ) by using a video-controlled tensile testing method [14].

Actually, the mechanical properties of polymers are a priori, pressure dependent [15]. This is another reason to investigate their tensile behavior. In terms of high strain sensitivity, the tensile properties have been reported for several polymers, for instance, polycarbonate [16-18], polyvinylchloride [19], polymethylmethacrylate [20], and epoxies [20-22], but not for PEEK. In this work, we are interested in assessing the tensile plastic behavior of PEEK for strain rates ranging from 0.0001 to $1000 / \mathrm{s}$. To the best of the authors' knowledge, this has never been reported before. In order to measure the true stress during plastic deformation, we aim also at measuring the PEEK's volume variation. For this purpose, digital image correlation (DIC) is applied. In addition, a constitutive equation will be proposed to take into account the sensitivity of the yield stress to the strain rate.

\section{Experimental Characterization}

2.1. Material and Specimens. The PEEK examined in this work is of grade $450 \mathrm{G}$. The DSC technique is a useful technique to obtain the characteristic temperatures and the crystallinity ratio [23]. Using a DSC analysis with a heating rate of $10^{\circ} \mathrm{C} / \mathrm{min}$ [9], the crystalline phase of PEEK has a melting point of $342^{\circ} \mathrm{C}$ whilst the glass transition temperature of its amorphous phase is equal to $152^{\circ} \mathrm{C}$. The ratio of the melting enthalpy to the theoretical melting enthalpy gives a crystallinity ratio of about $27 \%$.

Specimens were machined from rods of diameter $20 \mathrm{~mm}$, purchased from Ensinger France. The specimen geometry (Figure 1) is employed for both quasi-static and dynamic experiments. Therefore, the gauge length (middle part) is optimized in order to enhance the stress equilibrium at high strain rates, before yielding. The gauge length is therefore reduced to $L_{r s}=6 \mathrm{~mm}$.

Actually, it is hard to obtain stress homogeneity in the beginning of a dynamic test. However, one of the main objectives of this work is to investigate the yield behavior of PEEK. We have then designed the specimens in order to achieve the stress homogeneity before yielding.

A random speckle pattern is painted on the gauge zones in order to enable a digital image correlation analysis for fullfield strain measurements. Three experiments are undertaken for each strain rate.

2.2. Test Procedure. Specimens were tested at low strain rates using an Instron 5584 screw-driven testing machine. Experiments have been undertaken for crosshead velocities up to $36 \mathrm{~mm} / \mathrm{min}$. At quasi-static strain rates, the force is measured using an Instron 2525-171 $150 \mathrm{kN}$ load cell. The machine rigidity is neglected regarding the maximum force applied on specimens.

On the other hand, a tensile crossbow system [15, 24], sketched in Figure 2, is used for dynamic strain rates. The operating mode is similar to the compression crossbow system used in $[8,9,25]$. Actually, the crossbow technique is similar to the direct-impact Hopkinson bar setup except for the loading system [9]. Indeed, the projectile bar is now replaced by a rigid mass of $210 \mathrm{~kg}$. This rigid mass is composed from two parts (Figures 2(b) and 2(c)). The first part (Mass 1) is constructed from two pieces with dimensions of $220 \times 200 \times 250 \mathrm{~mm}^{3}$ each, distanced by $185 \mathrm{~mm}$. The space $(185 \mathrm{~mm})$ is occupied by a second mass (Mass 2 ) which has a $u$-form with dimensions of $45 \times 130 \times 115 \mathrm{~mm}^{3}$ and a spacing distance of $90 \mathrm{~mm}$. By the mean of elastic ropes, the guided rigid mass is accelerated up to a desired impact velocity. Changing the stiffness of ropes leads to different impact velocities. Before the test, the specimen is screwed from one end to a long elastic steel bar of $30 \mathrm{~mm}$ in diameter and $6000 \mathrm{~mm}$ in length. The second end of the specimen is screwed to a grip which has to receive the impact of the rigid mass. A zoom on the impact zone is presented in Figure 2(c) and schematized in Figure 3, including the dimensions of each piece. Figures 2 and 3 show a guiding device introduced in the tensile crossbow configuration in order to maintain the impact elements coaxial. Strain gauges, of type EA 06044 TP 350, provided by GAROS Groupe (France), are mounted in half Wheatstone bridge circuits and cemented in three locations of the bar. Using the signals of the elastic waves recorded by the gauges, the tensile force in the specimen can be determined.

The crossbow apparatus is outfitted with a high speed video camera of type Fastcam-ultima APX manufactured by Photron Limited, fitted with a high sensitivity imaging sensor enabling recording under extremely low level of light. The APX camera has a maximum recording speed of 120000 frames/s. For the highest speed tests, the acquisition frequency is 24000 frames/s, using a resolution of $512 * 128$ pixels. Lower rates are used for quasi-static experiments. To this aim, a frequency adaptor was connected to the rapid camera in order to acquire images at the desired low rate. 


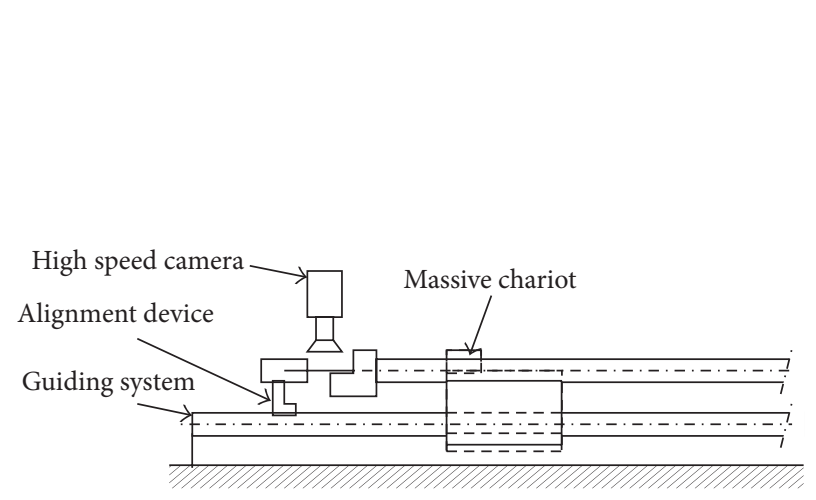

(a)

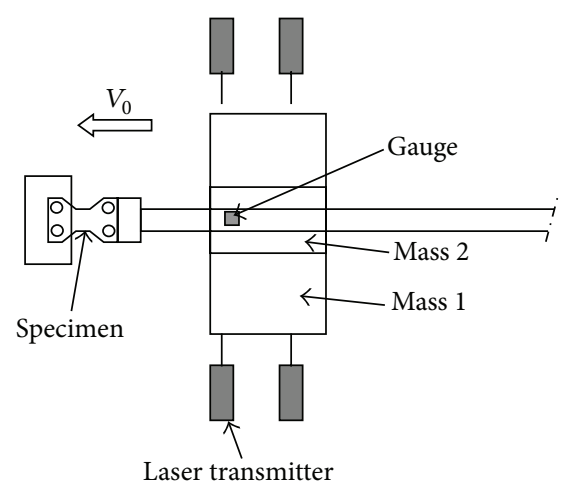

(b)

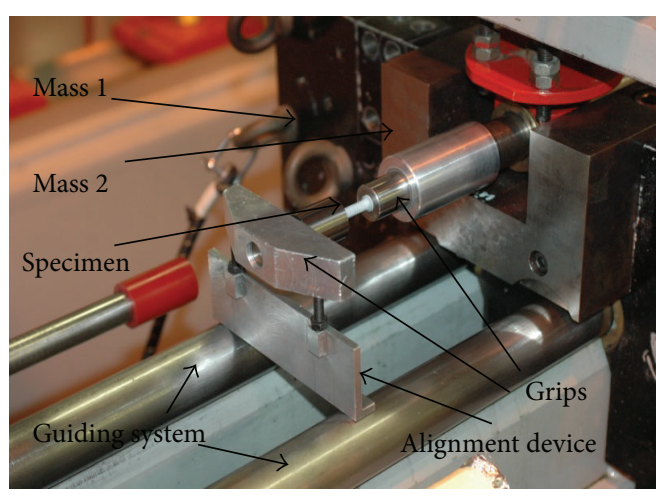

(c)

FIGURE 2: Crossbow system in tensile configuration: side view (a), top view (b), and overview (c).

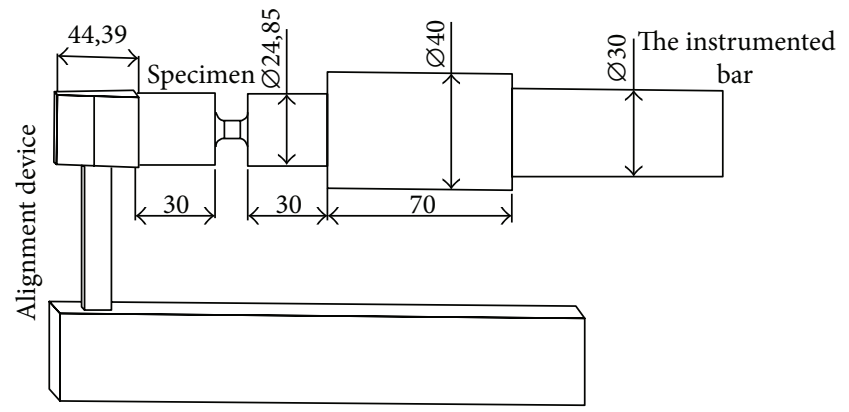

FIGURE 3: Impact system of the crossbow with the dimensions (in $\mathrm{mm}$ ) of each piece.

The tensile crossbow system works like a direct-impact Hopkinson bar machine. Thus the calibration of this system resembles the one used for the Hopkinson bar setup. More precisely, the long elastic bar is impacted by a shorter rod. The impact velocity can be determined by laser sensors. Subsequently, the calibration is carried out by writing that the impact kinetic energy of the striker rod is equal to the kinetic and deformation energy transmitted to the long bar.
2.3. Analysis. We are interested in this work to determine the uniaxial tensile behavior of PEEK. Therefore, we need to measure the true stress-true (logarithmic) strain curves. In terms of strain, the successive configurations of the specimen recorded during the experiment are analyzed by a DIC software, ICASOFT [26], developed by INSA Lyon, France. The main purpose is to compute the 2D-strain field. The grid used to mesh the specimens has a pattern size of $6 * 6$ pixels. The displacement-pixel conversion ratio is of $0.094 \mathrm{~mm} / \mathrm{pixel}$. The deformation is given in terms of the logarithmic strain field (Hencky strain). Subsequently, longitudinal and transversal strains were calculated using $2 \mathrm{~mm}$ long virtual strain gauges (Figure 4). The locations of these gauges are determined a posteriori and they are placed in the necking zone.

In addition to the axial true strain, we are also interested in determining the true axial stress. To this aim, an elementary cylindrical volume is virtually isolated (Figure 5). Subsequently, the axial and transverse strains, assumed homogeneous in this elementary volume, are considered. The transverse strain is used in calculating the change of the crosssectional area [15, 27-29]. Both transverse and axial strains are needed to calculate the volume change. 


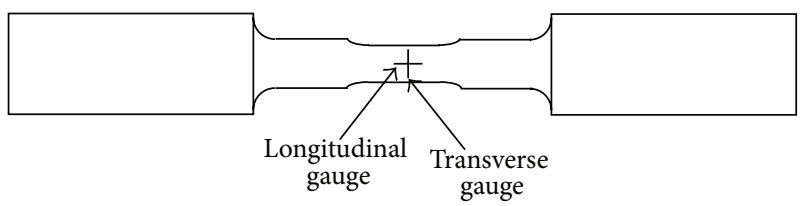

FIgURE 4: Positions of the virtual longitudinal and transversal strain gauges.

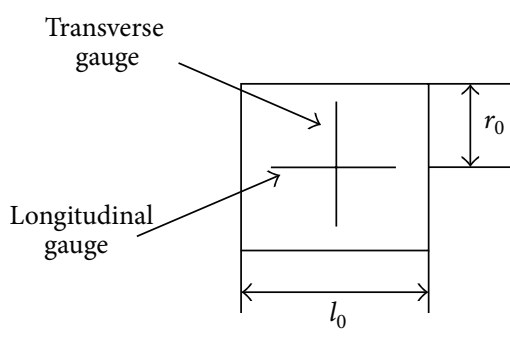

(a)

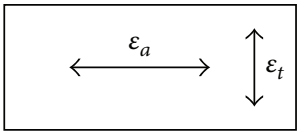

(b)
FIgURE 5: Elementary cylindrical volume: initial configuration (a) and deformed configuration (b).

Let $\varepsilon_{a}$ and $\varepsilon_{t}$ be the axial and transversal strains, respectively. They read

$$
\begin{aligned}
\varepsilon_{a} & =\ln \left(\frac{l}{l_{0}}\right), \\
\varepsilon_{t} & =\ln \left(\frac{r}{r_{0}}\right),
\end{aligned}
$$

where $l_{0}$ and $r_{0}$ are the initial length and radius, respectively, of the elementary volume. Likewise, $l$ and $r$ are the length and radius, respectively, of the deformed elementary volume. Writing the initial volume $V_{0}$ in terms of $l_{0}$ and $r_{0}$ and the volume after deformation $V$ in terms of $l$ and $r$, the volume ratio reads

$$
\rho_{V}=\frac{V}{V_{0}}=\exp \left(\varepsilon_{a}+2 \varepsilon_{t}\right)
$$

Similarly, the cross-sectional area ratio is given by

$$
\rho_{A}=\frac{A}{A_{0}}=\frac{\pi r^{2}}{\pi r_{0}^{2}}=\exp \left(2 \varepsilon_{t}\right),
$$

where $A_{0}$ and $A$ are the initial and current cross-sectional areas, respectively, of the elementary volume.

Once the cross-sectional area variation is computed, the true axial stress $\sigma_{a}$ can be determined by computing the ratio of the measured force $F$ to the current cross-sectional area; that is,

$$
\sigma_{a}=\frac{F}{A}=\frac{F}{\rho_{A} A_{0}} .
$$

The force is measured by the mean of a force transducer in quasi-static experiments, whereas it is deduced from the bar strain gauge's signal at high strain rate tests. For strain rates around $150 / \mathrm{s}$, a wave separation technique is used to separate waves propagating in opposite directions [30]. Once the true stress is determined, the tensile yield stress is calculated as the first maximum stress observed in the true stress-true strain curve.

\subsection{Results}

2.4.1. Deformation Process. A typical deformation process is depicted in Figure 6. Even though the images correspond to an experiment undertaken at $0.001 / \mathrm{s}$, the same deformation process occurs for different strain rates. In the beginning, the deformation is uniform along the central part, Figure 6(a). Upon the yielding, necking appears, Figure 6(b). Subsequently, this necking propagates and affects a more important zone of the specimen gauge zone, Figure 6(c). Finally, a significant crack appears somewhere in the neckingaffected zone. It propagates perpendicularly to the tensile loads till complete failure, Figure 6(d). The appearance of necking makes the stress and strain fields heterogeneous in the specimen. The DIC analysis is then focused on the necking zone in order to deduce a local strain measurement [31].

2.4.2. Volume Change. The volume ratio is plotted in Figure 7 for true axial strains up to $\sim 60 \%$. The same tendency is observed for the six studied strain rates. Namely, the volume ratio increases with an increasing strain. This means that the specimen undergoes an increasing dilatation. The PEEK's volume increases by $\sim 3.3,6.2$, and $16.2 \%$ when deformed by 10,20 , and $40 \%$, respectively. This is very close to the polycarbonate dilatation. Indeed, Hiermaier and Huberth [29] reported a $14-18 \%$ increase in volume at axial strain of $40 \%$. However, this is lower than the volume dilatation in polypropylene as reported in [15]. This can be explained by the differences in the texture of the three polymers. Indeed, Bai et al. [32], based on a microstructural study, concluded that the volume dilatation in polymers under tension is mainly due to a damaging mechanism. This process starts from the microdefaults within the polymer, more precisely, in the amorphous part. Subsequently, the deformation localizes around these defaults leading to an intense stress concentration. This ultimately yields the creation of voids and the increase of the polymer material volume [33]. In line with this, El-Qoubaa and Othman [8] reported that the volume of PEEK remains constant during uniaxial compression. This is mainly attributed to the fact that cracks are rather closed under compression and do not propagate, while it is the case under tension.

Elsewhere, most of the curves of Figure 7 have the same tendency; in other words, there is no clear influence of the strain rate on the volume change. Consequently, an average volume ratio, $\rho_{V \text {,avg }}=\left(V / V_{0}\right)_{\text {avg }}$, and an average crosssectional area ratio, $\rho_{A \text { avg }}=\left(A / A_{0}\right)_{\text {avg }}$, are calculated for PEEK from all tensile experiments, independently of the strain rate. These average ratios are plotted in Figure 8 in terms of the tensile axial strain. The average cross-sectional area ratio will be used in calculating the true stress knowing the applied force on the specimen as detailed in (4). 


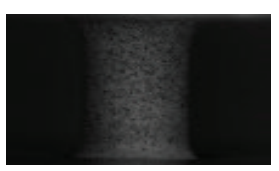

(a)

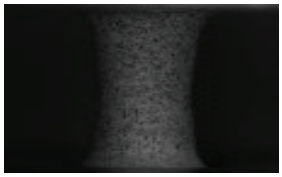

(b)

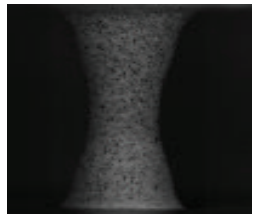

(c)

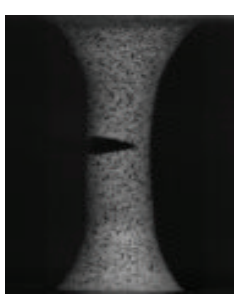

(d)

FIGURE 6: Deformation process during a 0.001/s strain rate experiment at (a) $19 \mathrm{~s}$, (b) $191 \mathrm{~s}$, (c) $530 \mathrm{~s}$, and (d) $1581 \mathrm{~s}$.

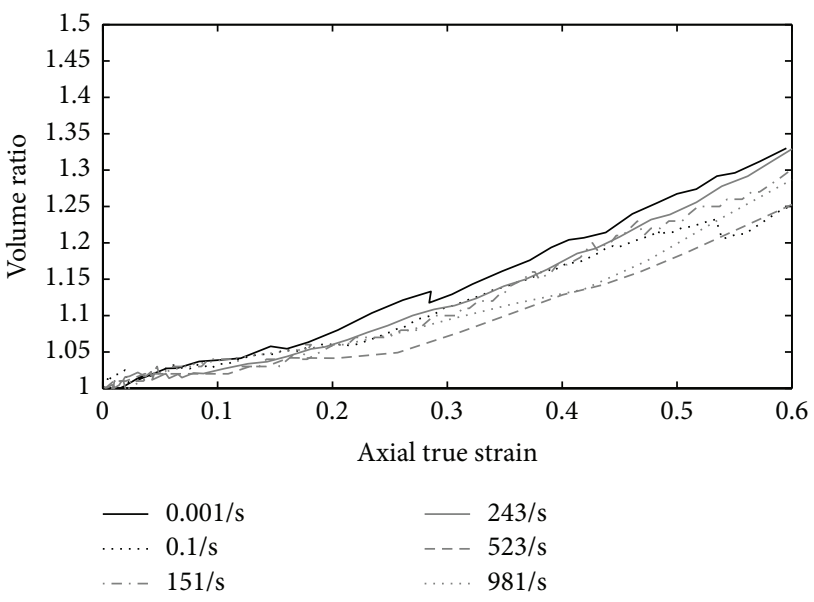

FIgURE 7: Volume change at several strain rates.

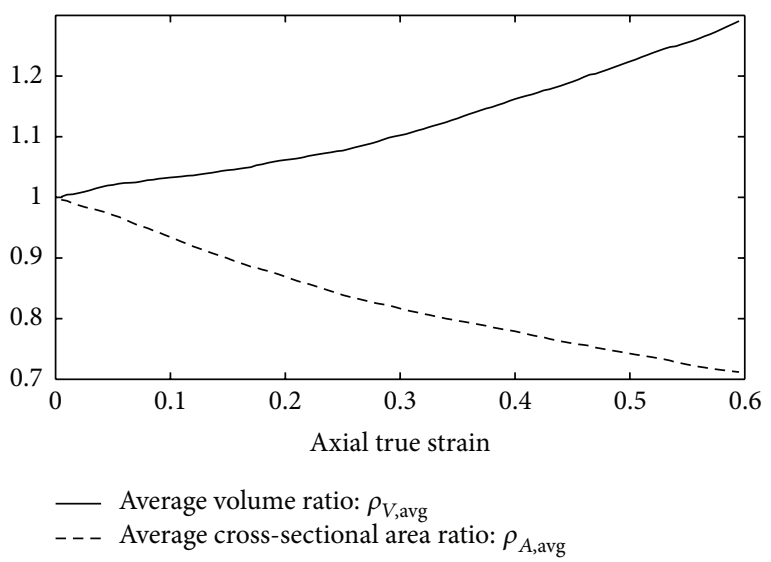

FIGURE 8: Average volume ratio and average cross-sectional area ratio in terms of the tensile axial strain.

2.4.3. Stress-Strain Curves. The true stress is determined by dividing the measured force by the cross-sectional area which is determined using the DIC analysis. The true strain is also obtained from the DIC analysis as well. The strain rate is therefore determined as the time derivative of the strain. The true stress-strain curves are depicted in Figure 9. The strain rate variations during quasi-static and dynamic experiments are represented in Figure 10.

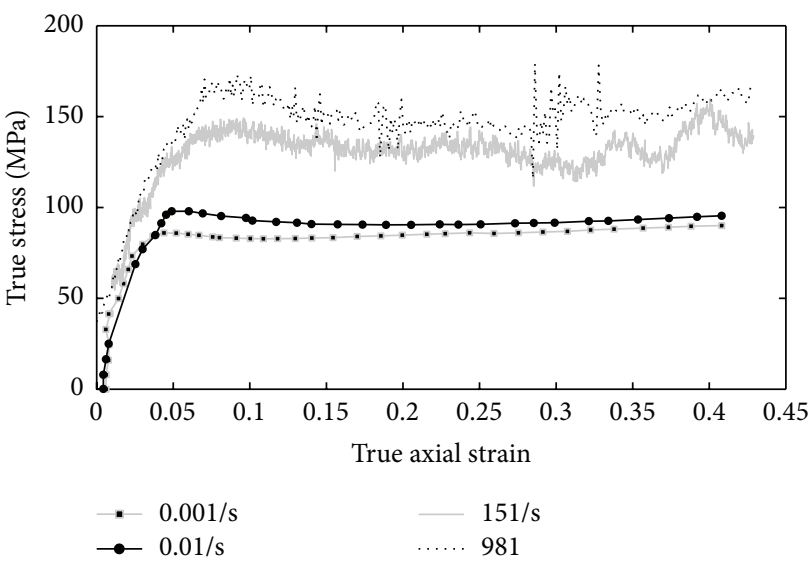

FIgURE 9: Stress-strain behavior at different strain rates.

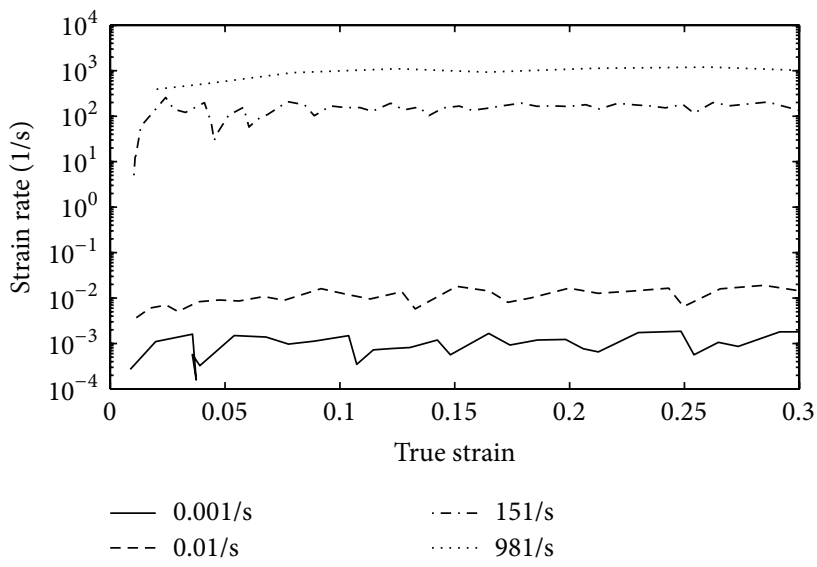

Figure 10: Strain rate variation during quasi-static and dynamic experiments.

In terms of stress-strain curves, the tendency is almost the same for the whole strain rate range. A rapid increase in the stress, for small strains, corresponding to the viscoelastic behavior of the PEEK is observed. A maximum stress is reached corresponding to the yield stress thereafter. It is then followed by softening till a minimum stress is reached. Therefore, the minimum stress is slightly lower than the yield (first maximum) stress.

In Section 2.1, we stated that the specimens are designed in order to achieve the stress homogeneity before yielding. 


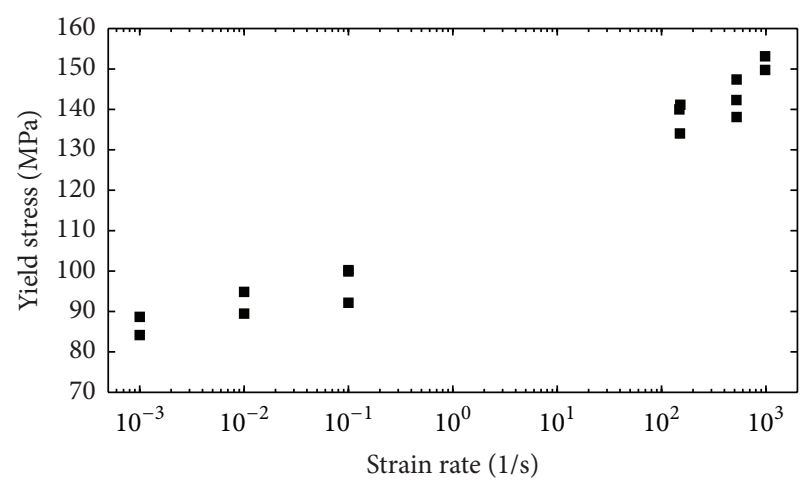

FIGURE 11: Sensitivity of the yield stress to strain rate.

The most difficult situation is encountered at the highest strain rate test, that is, $\sim 1000 / \mathrm{s}$. As the strain rate increases, it becomes harder to obtain stress homogeneity within the specimen. Let us consider Figure 9. At the strain rate nearly 981/s, the yielding happens at around $6.5 \%$ of strain. This corresponds to a time of $66 \mu \mathrm{s}$. During this time, the tensile wave, which is moving with a velocity of $1700 \mathrm{~m} / \mathrm{s}$, can travel a distance around $115 \mathrm{~mm}$ within the specimen. Obviously, this distance is much higher than the gauge length. This means that the tensile wave makes several round-trips in the gauge part of the specimen. This provides the establishment of the stress homogeneity in the specimen. Indeed, Koubaa et al. [34] showed that if the tensile wave travels by nearly three times the gauge length, the stress homogeneity is ensured.

2.4.4. Yield Stress. Figure 11 shows the tensile yield stress as a function of the strain rate. At each strain rate, the yield stress is measured at three experiments and is taken as the first maximum stress. The tensile yield stress of PEEK is highly sensitive to the strain rate. Namely, it increases if strain rate increases (Figure 11). The yield stress at $~ 1000 / s$ is $~ 84 \%$ higher than the one at $0.001 / \mathrm{s}$. This gives an average increase of $11.67 \mathrm{MPa}$ /decade. However, the strain rate sensitivity is much higher at high strain rates. This is the most reported behavior in the literature for polymers [9]. In the subsequent section, this behavior will be compared to the Ree-Eyring constitutive model and to a new model proposed in this work.

\section{Constitutive Equation for the Yield Stress}

3.1. Constitutive Equation. In the previous section, the yield stress of PEEK is determined over a wide strain rate range. Garcia-Gonzalez et al. [1] used the Johnson-Cook model [3537], which is already preimplemented in several finite element codes, to fit the yield stress behavior. However, the strain rate sensitivity of yield stress increases at high strain rates. This behavior can be modeled using the power-law equation. Alternatively, Ree and Eyring [38] have suggested the use of multiple relaxation processes.

The Ree-Eyring equation is a generalization, by using two relaxation processes, of the original equation of Eyring [39], which considers the yielding as a thermal activated process. By considering two relaxation processes, the yield stress predicted by the Ree-Eyring equation reads

$$
\begin{aligned}
\frac{\sigma_{\mathrm{RE}}}{T}= & A_{\alpha}\left(\ln \left(2 C_{\alpha} \dot{\varepsilon}\right)+\frac{Q_{\alpha}}{R T}\right) \\
& +A_{\beta} \sinh ^{-1}\left(C_{\beta} \dot{\varepsilon} \exp \left(\frac{Q_{\beta}}{R T}\right)\right),
\end{aligned}
$$

where $A_{\alpha}, A_{\beta}, C_{\alpha}$, and $C_{\beta}$ are four material constants, $Q_{\alpha}$ and $Q_{\beta}$ are the activation energies of the two relaxation processes, $R$ is the universal gas constant, and $T$ is the absolute temperature. For identification purpose, (5) is simplified to

$$
\sigma_{\mathrm{RE}}=a_{\alpha}+b_{\alpha} \ln (\dot{\varepsilon})+b_{\beta} \sinh ^{-1}\left(c_{\beta} \dot{\varepsilon}\right),
$$

where $a_{\alpha}, b_{\alpha}, b_{\beta}$, and $c_{\beta}$ are four real constants.

Recently, the authors [9] have proposed a modified Eyring equation. It assumes an apparent activation volume that decreases with strain rate [40-42]. The apparent activation volume is proportional to the slope of the logarithm of the strain rate in terms of yield stress [43]. More precisely, the apparent activation volume, $V^{*}$, is defined as

$$
V^{*}=k_{B} T\left(\frac{\partial \ln \dot{\varepsilon}}{\partial \sigma_{y}}\right)_{T, P},
$$

where $k_{B}, \sigma_{y}$, and $\dot{\varepsilon}$ are the Boltzmann constant, yield stress, and strain rate, respectively. Figure 10 indicates that the slope of the yield stress curve in terms of strain rate increases if the strain rate increases, suggesting that the activation volume is decreasing with increased strain.

In terms of the original Eyring model, the yield stress reads

$$
\sigma=\sigma_{0}+\frac{k_{B} T}{V_{0}} \ln (\dot{\varepsilon}),
$$

where $\sigma_{0}$ is a characteristic stress and $V_{0}$ is the activation volume. This suggests that the activation volume for each relaxation process, assumed constant, is given by

$$
V_{\alpha, \beta}=\frac{k_{B} T}{b_{\alpha, \beta}}
$$

where $V_{\alpha}$ and $V_{\beta}$ are the activation volumes of the relaxation processes $\alpha$ and $\beta$.

Instead of using a constant activation volume for each process, the authors [9] have proposed using an apparent activation volume that decreases with strain rate. More precisely, the apparent activation volume writes [9]

$$
V^{*}=V_{0} \exp \left(-\sqrt{\frac{\dot{\varepsilon}}{\dot{\varepsilon}_{c}}}\right),
$$

where $\dot{\varepsilon}_{c}$ stands for a critical strain rate. The modified Eyring constitutive equation is then written as

$$
\sigma_{\mathrm{ME}}=\sigma_{0}+\frac{k_{B} T}{V_{0}} \exp \left(\sqrt{\frac{\dot{\varepsilon}}{\dot{\varepsilon}_{c}}}\right) \ln \left(\frac{\dot{\varepsilon}}{\dot{\varepsilon}_{0}}\right),
$$


where $\sigma_{\mathrm{ME}}$ holds for the yield stress predicted by the new Eyring-based model and $\dot{\varepsilon}_{0}=1 / \mathrm{s}$ is a normalizing strain rate. $\sigma_{\mathrm{ME}}$ depends herein on three parameters: $V_{0}, \dot{\varepsilon}_{c}$, and $\sigma_{0} . \sigma_{0}$ is the yield stress for a strain rate equal to $1 / \mathrm{s} . V_{0}$ can be assimilated to the activation volume of the first Eyring process; that is, $V_{0} \approx V_{\alpha}$. The critical strain rate $\dot{\varepsilon}_{c}$ gives the strain rate range where only one Eyring process is activated. Put in other words, $\exp \left(-\sqrt{\dot{\varepsilon} / \dot{\varepsilon}_{c}}\right) \approx 1$ for strain rates $\dot{\varepsilon} \ll \dot{\varepsilon}_{c}$. This suggests that (8) and (11) will converge for strain rates $\dot{\varepsilon} \ll \dot{\varepsilon}_{c}$.

3.2. Identification. The identification procedure aims at finding the best set of materials constants that minimizes the discrepancy between the measured yield stress and the yield stress predicted by both the Ree-Eyring (5) and the new modified Eyring constitutive equation (11). Let $\Sigma_{y}$ and $\Sigma_{\chi}$ be the experimentally measured yield stresses, which can be arranged in vectors, and the corresponding yield stress vector computed by using the constitutive equation $\chi$, respectively, where $\chi=\mathrm{RE}$ for the Ree-Eyring equation and $\chi=\mathrm{ME}$ for the new proposed equation.

The minimization procedure depends on the definition of the cost function. In order to take into account the local and global errors [9], the cost function is defined as the average of the maximum and the Euclidean distances between the experimental and the model-predicted yield stresses. Put in other words, the cost function can be written as

$$
f_{\chi}\left(\kappa_{1}^{\chi}, \kappa_{2}^{\chi}, \ldots\right)=\frac{1}{2}\left(\operatorname{EucErr}_{\chi}+\operatorname{MaxErr}_{\chi}\right)
$$

where $\kappa_{1}^{\chi}, \kappa_{2}^{\chi}, \ldots$ are the material constants defining the constitutive equation $\chi$ and $\operatorname{EucErr}_{\chi}$ and $\operatorname{MaxErr}_{\chi}$ are the Euclidean and the maximum relative distances that write

$$
\begin{aligned}
\operatorname{EucErr}_{\chi} & =\frac{\left\|\Sigma_{\chi}-\widehat{\Sigma}\right\|_{2}}{\|\widehat{\Sigma}\|_{2}}, \\
\operatorname{MaxErr}_{\chi} & =\frac{\left\|\Sigma_{\chi}-\widehat{\Sigma}\right\|_{\infty}}{\|\widehat{\Sigma}\|_{\infty}} .
\end{aligned}
$$

Respectively, \|\|$_{2}$ defines the Euclidean distance and \|\|$_{\infty}$ denotes the maximum distance.

The minimization of the cost function $f_{\mathrm{RE}}$ yields the four material constants, $a_{\alpha}, b_{\alpha}, b_{\beta}$, and $c_{\beta}$, of (6). Subsequently, the activation volumes of $\alpha$ and $\beta$ processes are calculated using (9); that is, $V_{\alpha}=k_{B} T / b_{\alpha}$ and $V_{\beta}=k_{B} T / b_{\beta}$, respectively. Likewise, the minimization of the cost function $f_{\mathrm{ME}}$ gives the three material constants of the newly proposed modified Eyring equation, that is, $V_{0}, \dot{\varepsilon}_{c}$, and $\sigma_{0}$.

3.3. Results and Discussion. The identification procedure is carried out for both the Ree-Eyring and the new modified Eyring equations. The material constants of the Ree-Eyring equation are given in Table 1 , whereas the material constants of the new model are given in Table 2. The Ree-Eyring model gives a first-process activation volume and a second-process activation volume of $V_{\alpha}=8.42 \times 10^{-28} \mathrm{~m}^{3}$ and $V_{\beta}=8.79 \times$ $10^{-32} \mathrm{~m}^{3}$, respectively. The new Eyring-based model gives

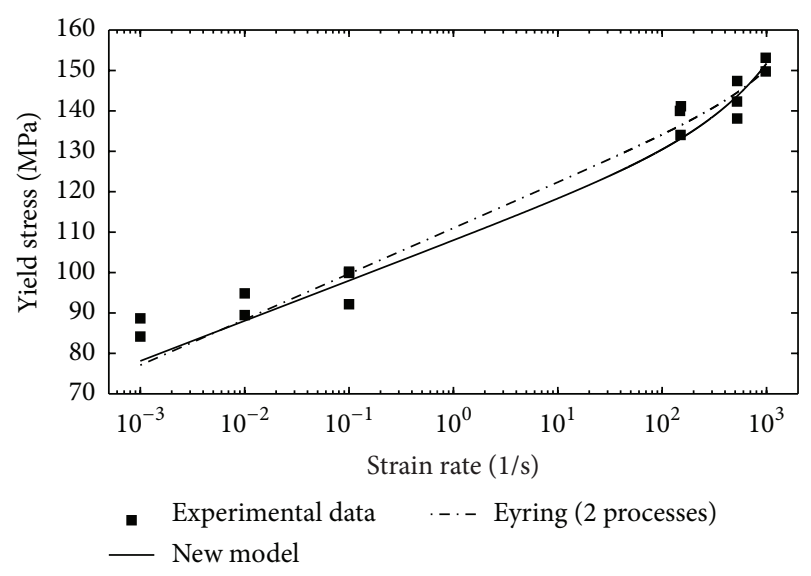

FIGURE 12: Identification of the yield stress using the Ree-Eyring model and the modified Eyring model.

TABLE 1: Material constants of Ree-Eyring equation.

\begin{tabular}{lccc}
\hline$a_{\alpha}(\mathrm{Pa})$ & $b_{\alpha}(\mathrm{Pa})$ & $b_{\beta}(\mathrm{Pa})$ & $c_{\beta}(\mathrm{s})$ \\
\hline $1.11 \times 10^{8}$ & $4.91 \times 10^{6}$ & $4.60 \times 10^{10}$ & $1.17 \times 10^{-7}$ \\
\hline
\end{tabular}

TABLE 2: Material constants of the new model.

\begin{tabular}{lccc}
\hline & $\sigma_{0}(\mathrm{~Pa})$ & $V_{0}\left(\mathrm{~m}^{3}\right)$ & $\dot{\varepsilon}_{c}\left(\mathrm{~s}^{-1}\right)$ \\
\hline Tensile & $1.08 \times 10^{8}$ & $9.42 \times 10^{-28}$ & $6.87 \times 10^{3}$ \\
\hline
\end{tabular}

TABLE 3: Average error and correlation coefficient.

\begin{tabular}{lcc}
\hline & Error (\%) & Correlation coefficient \\
\hline Ree-Eyring model & 5.8 & 0.98 \\
Eyring-based model & 5.1 & 0.98 \\
\hline
\end{tabular}

$V_{0}=9.42 \times 10^{-28} \mathrm{~m}^{3}$ while the other parameters are $\sigma_{0}=$ $108 \mathrm{MPa}$ and $\dot{\varepsilon}_{c}=6870 / \mathrm{s}$ for the characteristic stress and the critical strain rate, respectively. It is worth noting that $V_{0}$ is close to $V_{\alpha}$ as expected. Likewise, $\sigma_{0}$ is almost equal to $a_{\alpha}=111 \mathrm{MPa}$ as these constants interpret the yield stress at a strain rate of $1 / \mathrm{s}$. Table 2 reports also that the critical strain rate in tension is equal to $6870 / \mathrm{s}$. This suggests that the transition in strain rate sensitivity occurs at around 69/s in tension.

The Ree-Eyring model and the new modified Eyring equation fit well the experimental data (Figure 12). Their relative errors are $5.8 \%$ and $5.1 \%$, respectively, whereas the correlation coefficients obtained by the two models are both about 0.98 (Table 3 ). Thus, the two equations give almost the same approximation. However, the new model uses only three material constants.

\section{Conclusion}

The tensile mechanical behavior of PEEK was characterized over a large domain of strain rates (from 0.001 to 1000/s) using a conventional tensile machine and a customer-designed tensile crossbow machine. The digital image correlation technique was used together with a high speed video camera 
to determine the volume change. It was depicted that PEEK undergoes an increasing volume dilatation while axial tensile strain increases. Moreover, this volume change is showed to be strain rate independent. Thus, an average volume change is computed and used to determine the true stress-true strain behavior. It is then reported that the mechanical behavior is highly dependent on the strain rate. Namely, the yield stress is increasing with the increase of strain rate. Besides, the strain rate sensitivity is more important at higher strain rates. A new constitutive equation was then proposed to fit this behavior. This constitutive model is almost as accurate as the Ree-Eyring model, though only three material constants are used.

\section{Conflict of Interests}

The authors declare that there is no conflict of interests regarding the publication of this paper.

\section{References}

[1] D. Garcia-Gonzalez, A. Rusinek, T. Jankowiak, and A. Arias, "Mechanical impact behavior of polyether-ether-ketone (PEEK)," Composite Structures, vol. 124, pp. 88-99, 2015.

[2] H. Xu, Y. Wang, X. Su, X. Zhang, and X. Zhang, "Safety and efficiency of biomimetic nanohydroxyapatite/polyamide $66 \mathrm{com}$ posite in rabbits and primary use in anterior cervical discectomy and fusion," International Journal of Polymer Science, vol. 2014, Article ID 696045, 6 pages, 2014.

[3] S. M. Walley and J. E. Field, "Strain rate sensitivity of polymers in compression from low to high rates," DYMAT Journal, vol. 1, pp. 211-227, 1994.

[4] S. Hamdan and G. M. Swallowe, "The strain-rate and temperature dependence of the mechanical properties of polyetherketone and polyetheretherketone," Journal of Materials Science, vol. 31, no. 6, pp. 1415-1423, 1996.

[5] S. Hamdan and G. M. Swallowe, "Crystallinity in PEEK and PEK after mechanical testing and its dependence on strain rate and temperature," Journal of Polymer Science, Part B: Polymer Physics, vol. 34, no. 4, pp. 699-705, 1996.

[6] G. M. Swallowe, J. O. Fernandez, and S. Hamdan, "Crystallinity increases in semi crystalline polymers during high rate testing," Journal de Physique IV France C3, vol. 7, pp. 453-458, 1997.

[7] P. J. Rae, E. N. Brown, and E. B. Orler, "The mechanical properties of poly(ether-ether-ketone) (PEEK) with emphasis on the large compressive strain response," Polymer, vol. 48, no. 2, pp. 598-615, 2007.

[8] Z. El-Qoubaa and R. Othman, "Volume change in polyetheretherketone under compression loads over wide ranges of strain rate and temperature," Journal of Strain Analysis for Engineering Design, vol. 49, no. 5, pp. 315-324, 2014.

[9] Z. El-Qoubaa and R. Othman, "Characterization and modeling of the strain rate sensitivity of polyetheretherketone's compressive yield stress," Materials \& Design, vol. 66, pp. 336-345, 2015.

[10] M. F. Talbott, G. S. Springer, and L. A. Berglund, "The effects of crystallinity on the mechanical properties of PEEK polymer and graphite fiber reinforced PEEK," Journal of Composite Materials, vol. 21, no. 11, pp. 1056-1081, 1987.

[11] W. I. Lee, M. F. Talbott, G. S. Springer, and L. A. Berglund, "Effects of cooling rate on the crystallinity and mechanical properties of thermoplastic composites," Journal of Reinforced Plastics and Composites, vol. 6, no. 1, pp. 2-12, 1987.

[12] L. H. Lee, J. J. Vanselow, and N. S. Schneider, "Effects of mechanical drawing on the structure and properties of PEEK," Polymer Engineering and Science, vol. 28, no. 3, pp. 181-187, 1988.

[13] C. G'sell and A. Dahoun, "Evolution of microstructure in semicrystalline polymers under large plastic deformation," Materials Science and Engineering A, vol. 175, no. 1-2, pp. 183-199, 1994.

[14] C. G'Sell, J. M. Hiver, A. Dahoun, and A. Souahi, "Videocontrolled tensile testing of polymers and metals beyond the necking point," Journal of Materials Science, vol. 27, no. 18, pp. 5031-5039, 1992.

[15] V. Delhaye, A. H. Clausen, F. Moussy, R. Othman, and O. S. Hopperstad, "Influence of stress state and strain rate on the behaviour of a rubber-particle reinforced polypropylene," International Journal of Impact Engineering, vol. 38, no. 4, pp. 208218, 2011.

[16] S. S. Sarva and M. C. Boyce, "Mechanics of polycarbonate during high-rate tension," Journal of Mechanics of Materials and Structures, vol. 2, no. 10, pp. 1853-1880, 2007.

[17] Z. N. Yin and T. J. Wang, "Deformation response and constitutive modeling of PC, ABS and PC/ABS alloys under impact tensile loading," Materials Science and Engineering A, vol. 527, no. 6, pp. 1461-1468, 2010.

[18] K. Cao, Y. Wang, and Y. Wang, "Effects of strain rate and temperature on the tension behavior of polycarbonate," Materials and Design, vol. 38, pp. 53-58, 2012.

[19] J. L. Jordan, C. R. Siviour, and B. T. Woodworth, "High strain rate tensile and compressive effects in glassy polymers," EPJ Web of Conferences, vol. 26, Article ID 01001, 2012.

[20] W. Chen, F. Lu, and M. Cheng, "Tension and compression tests of two polymers under quasi-static and dynamic loading," Polymer Testing, vol. 21, no. 2, pp. 113-121, 2002.

[21] R. Gerlach, C. R. Siviour, N. Petrinic, and J. Wiegand, "Experimental characterisation and constitutive modelling of RTM-6 resin under impact loading," Polymer, vol. 49, no. 11, pp. $2728-$ 2737, 2008.

[22] E. A. Gültürk, M. Güden, and A. Taşdemirci, "Calcined and natural frustules filled epoxy matrices: the effect of volume fraction on the tensile and compression behavior," Composites Part B: Engineering, vol. 44, no. 1, pp. 491-500, 2013.

[23] M. Rahaman, M. A. Parvez, J. B. P. Soares, and I. A. Hussein, "Effect of polymerization conditions on thermal and mechanical properties of ethylene/1-butene copolymer made with Ziegler-Natta catalysts," International Journal of Polymer Science, vol. 2014, Article ID 654260, 10 pages, 2014.

[24] A. Martin, R. Othman, and P. Rozycki, "Experimental investigation of quasi-static and intermediate strain rate behaviour of polypropylene glass fibre (PPGF) woven composite," Plastics, Rubber and Composites, vol. 44, no. 1, pp. 1-10, 2015.

[25] S. Hamdan and G. M. Swallowe, "A crossbow system for high-strain-rate mechanical testing," Measurement Science and Technology, vol. 7, no. 7, pp. 1068-1072, 1996.

[26] http://icasoft.insa-lyon.fr/.

[27] S. Aloui, R. Othman, E. Verron, and P. Guégan, "Semi-analytic inverse method for rubber testing at high strain rates," Mechanics Research Communications, vol. 47, pp. 97-101, 2013.

[28] C. G'Sell, S.-L. Bai, and J.-M. Hiver, "Polypropylene/polyamide 6/polyethylene-octene elastomer blends. Part 2: volume dilatation during plastic deformation under uniaxial tension," Polymer, vol. 45, no. 17, pp. 5785-5792, 2004. 
[29] S. Hiermaier and F. Huberth, "Volume dilatation in a polycarbonate blend at varying strain rates," European Physical Journal: Special Topics, vol. 206, no. 1, pp. 173-181, 2012.

[30] R. Othman, P. Guégan, G. Challita, D. Lebreton, and F. Pasco, "A modified servo-hydraulic machine for testing at intermediate strain rates," International Journal of Impact Engineering, vol. 36, no. 3, pp. 460-467, 2009.

[31] F. Lauro, B. Bennani, D. Morin, and A. F. Epee, "The SEE method for determination of behaviour laws for strain rate dependent material: application to polymer material," International Journal of Impact Engineering, vol. 37, no. 6, pp. 715-722, 2010.

[32] S.-L. Bai, C. G'Sell, J.-M. Hiver, and C. Mathieu, "Polypropylene/polyamide 6/polyethylene-octene elastomer blends. Part 3. Mechanisms of volume dilatation during plastic deformation under uniaxial tension," Polymer, vol. 46, no. 17, pp. 6437-6446, 2005.

[33] A. Pawlak and A. Galeski, "Cavitation during tensile deformation of polypropylene," Macromolecules, vol. 41, no. 8, pp. 28392851, 2008.

[34] S. Koubaa, R. Othman, B. Zouari, and S. El-Borgi, "Finiteelement analysis of errors on stress and strain measurements in dynamic tensile testing of low-ductile materials," Computers and Structures, vol. 89, no. 1-2, pp. 78-90, 2011.

[35] F. Li, X. Qi, and D. Xiang, "Finite element modeling of crack generation in laser shock peening processed airfoils," Advances in Materials Science and Engineering, vol. 2014, Article ID 812705, 10 pages, 2014.

[36] E. Etemadi, J. Zamani Ashani, and M. V. Mousavi, "High strain rate and plastic deformation response of OFHC copper by finite element method," Advances in Mechanical Engineering, vol. 2014, Article ID 620854, 10 pages, 2014.

[37] G. Ljustina, M. Fagerström, and R. Larsson, "Rate sensitive continuum damage models and mesh dependence in finite element analyses," The Scientific World Journal, vol. 2014, Article ID 260571, 8 pages, 2014.

[38] T. Ree and H. Eyring, "The relaxation theory of transport phenomena," in Rheology: Theory and Applications, F. R. Eirich, Ed., vol. 2, chapter 3, Academic Press, New York, NY, USA, 1969.

[39] H. Eyring, "Viscosity, plasticity, and diffusion as examples of absolute reaction rates," The Journal of Chemical Physics, vol. 4, no. 4, pp. 283-291, 1936.

[40] M. F. Omar, H. M. Akil, and Z. A. Ahmad, "Measurement and prediction of compressive properties of polymers at high strain rate loading," Materials and Design, vol. 32, no. 8-9, pp. 42074215, 2011.

[41] M. F. Omar, H. M. Akil, Z. A. Ahmad, and S. Mahmud, "Particle size-dependent on the static and dynamic compression properties of polypropylene/silica composites," Materials and Design, vol. 45, pp. 539-547, 2013.

[42] M. F. Omar, H. M. Akil, Z. A. Ahmad, and S. Mahmud, "Static and dynamic compressive properties of polypropylene/zinc oxide nanocomposites," Polymer Engineering and Science, vol. 54, no. 4, pp. 949-960, 2014.

[43] J. Richeton, S. Ahzi, and L. Daridon, “Thermodynamic investigation of yield-stress models for amorphous polymers," Philosophical Magazine, vol. 87, no. 24, pp. 3629-3643, 2007. 

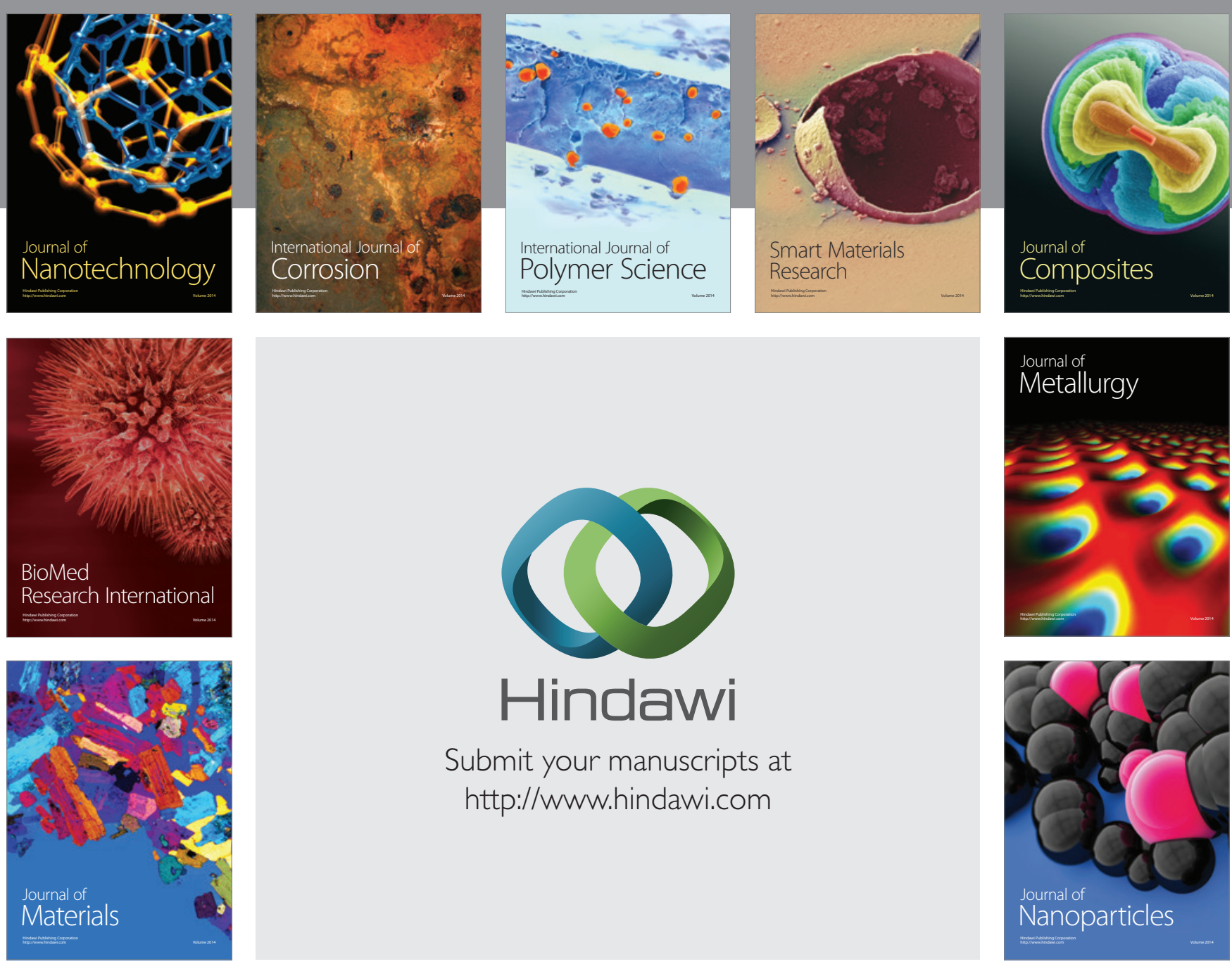

Submit your manuscripts at http://www.hindawi.com
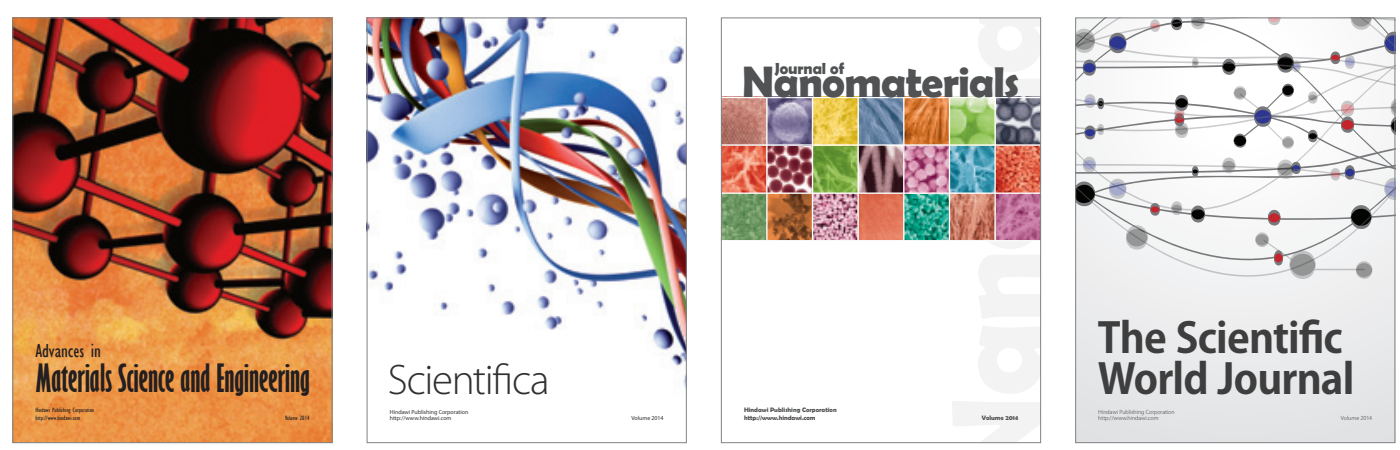

\section{The Scientific World Journal}
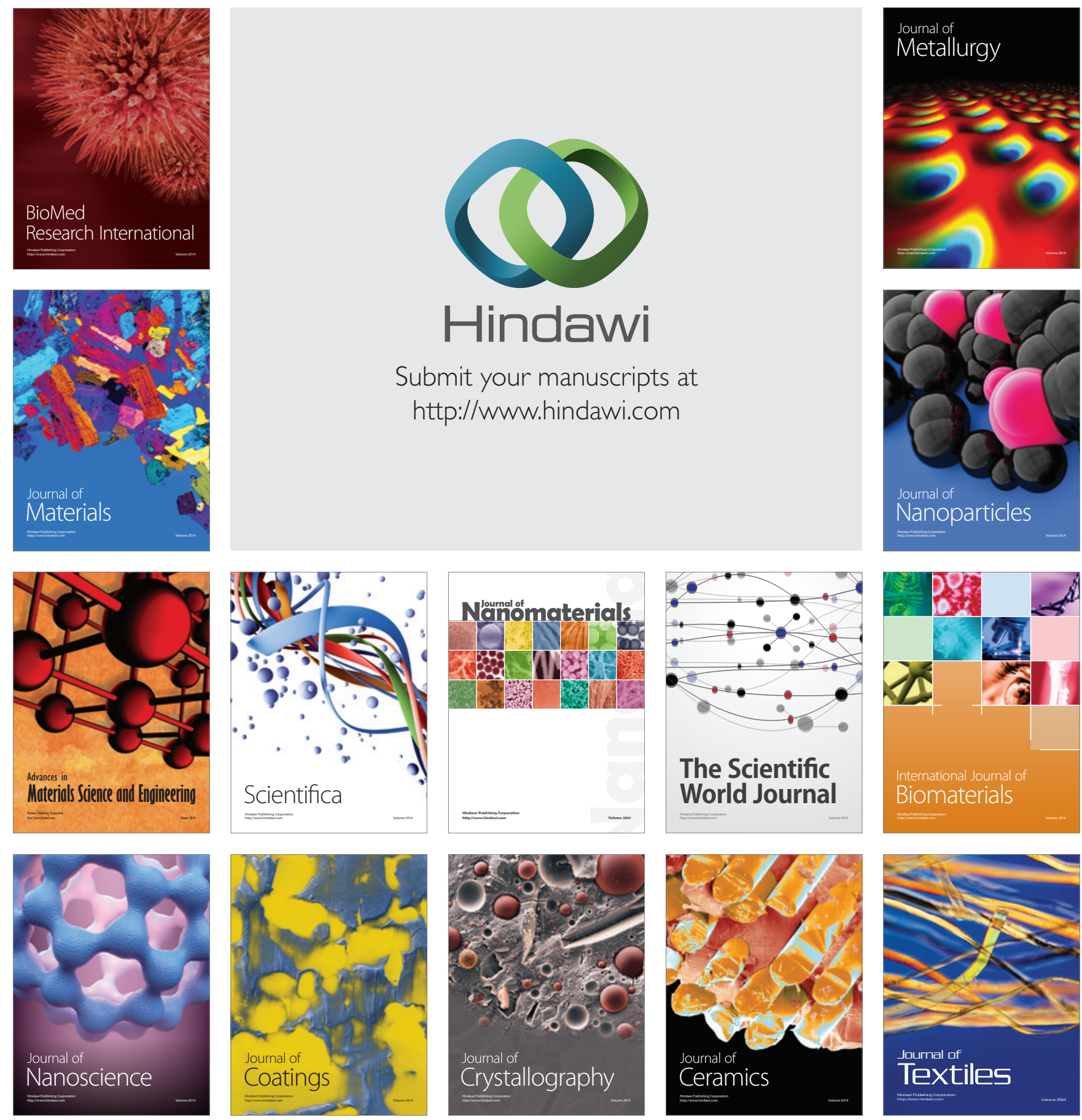